\begin{tabular}{|c|c|c|c|c|c|c|}
\hline \multirow{4}{*}{ Impact Factor: } & ISRA (India) & $=3.117$ & SIS (USA) & $=0.912$ & ICV (Poland) & $=6.630$ \\
\hline & ISI (Dubai, UAE & $=0.829$ & РИНЦ (Russia & $=0.156$ & PIF (India) & $=1.940$ \\
\hline & GIF (Australia) & $=0.564$ & ESJI (KZ) & $=8.716$ & IBI (India) & $=4.260$ \\
\hline & JIF & $=1.500$ & SJIF (Morocco & $=5.667$ & OAJI (USA) & $=0.350$ \\
\hline
\end{tabular}

\section{SOI: $1.1 /$ TAS DOI: $10.15863 /$ TAS International Scientific Journal Theoretical \& Applied Science}

\author{
p-ISSN: 2308-4944 (print) e-ISSN: 2409-0085 (online) \\ Year: 2019 Issue: $04 \quad$ Volume: 72
}

Published: $30.04 .2019 \quad$ http://T-Science.org
QR - Issue

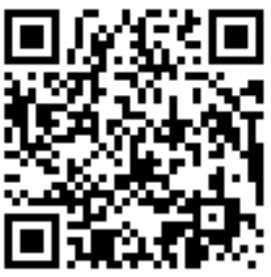

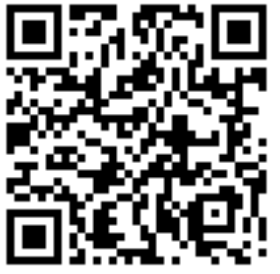

Unona Krahmaleva

Candidate of Science

Taraz State University named M.H.Dulaty

Vyacheslav Shevtsov graduate student of the 2nd course of the specialty "Mathematics»

Taraz State University named M.H.Dulaty

\title{
ANALYTICAL SOLUTION OF THE REGULAR PROBLEM OF THE STURM - LIOUVILLE PROBLEM IN MAPLE ENVIRONMENT
}

Abstract: To work with differential equations in Maple 17 has a fairly extensive set of tools. But despite this, the search for solutions to some differential equations is quite a difficult task, which requires knowledge of mathematical methods in this area. Given the fact that SCM have a developed programming language that contains procedural programming tools, it is possible to develop universal algorithms with which you can program the construction of solutions to such differential equations that will be used for specific physical problems.

Key words: Maple, differential equations, physical problems.

Language: Russian

Citation: Krahmaleva, U., \& Shevtsov, V. (2019). Analytical solution of the regular problem of the Sturm Liouville problem in maple environment. ISJ Theoretical \& Applied Science, 04 (72), 595-598.

Soi: http://s-o-i.org/1.1/TAS-04-72-84 Doi: croskef https://dx.doi.org/10.15863/TAS.2019.04.72.84

\section{АНАЛИТИЧЕСКОЕ РЕШЕНИЕ РЕГУЛЯРНОЙ ЗАДАЧИ ШТУРМА- ЛИУВИЛЛЯ В СРЕДЕ МАРLЕ}

Аннотация: Для работы с дифференциальными уравнениями в Maple 17 имеется достаточно обширный набор инструментов. Но несмотря на это поиск решения некоторых дифференциильных уравнений является достаточно сложной задачей, которая требует знания математических методов 8 данной области. Учитывая, тот факт, что СКМ имеют развитый язык программирования, который содержит средства прочедурного программирования, имеется возможность разрабатьвать универсальные алгоритмы с помощьью которых можно программировать построение решения таких дифференциальных уравнений, которые будут использованы для конкретных физических задач.

Ключевые слова: Мапл, дифференциальные уравнения, физические задачи.

\section{Introduction}

Рассмотрим обыкновенное однородное дифференциальное уравнение 2-го порядка:

$$
Y^{\prime \prime}(x)+\lambda Y(x)=0 \quad, a<x<b
$$

где $\lambda$ - параметр, принимающий любые значения.

Как известно, нахождение решения уравнения (1), которые удовлетворяют однородным линейным граничным условиям, заданные на концах интервала $(a, b)$ является задачей Штурма - Лиувилля. Нас интересует случай, когда интервал конечен, т.е. регулярная задача Штурма - Лиувилля, которая определена следующими граничными условиями:

$$
\begin{gathered}
Y(a)=0, Y(b)=0, \\
Y^{\prime}(a)=0, Y^{\prime}(b)=0,
\end{gathered}
$$

где (2) - граничные условия первого рода, (3)граничные условия второго рода.

Для нахождения решения рассматриваемой задачи необходимо определить те значения $\lambda$, при которых существуют нетривиальные решения, которые называются собственными функциями задачи. При этом, значения параметра 


\begin{tabular}{|c|c|c|c|c|c|c|}
\hline \multirow{4}{*}{ Impact Factor: } & ISRA (India) & $=3.117$ & SIS (USA) & $=0.912$ & ICV (Poland) & $=6.630$ \\
\hline & ISI (Dubai, UAE & $=0.829$ & РИНЦ (Russia & $=\mathbf{0 . 1 5 6}$ & PIF (India) & $=1.940$ \\
\hline & GIF (Australia) & $=0.564$ & ESJI (KZ) & $=8.716$ & IBI (India) & $=4.260$ \\
\hline & JIF & $=1.500$ & SJIF (Morocco & $=5.667$ & OAJI (USA) & $=0.350$ \\
\hline
\end{tabular}

$\lambda$ называются собственными значениями задачи.

\section{Materials and Methods}

Для нахождения аналитического решения задачи Штурма- Лиувилля

$$
\left\{\begin{array}{l}
Y^{\prime \prime}(x)+\lambda Y(x)=0 \\
Y(a)=Y(b)=0
\end{array}\right.
$$

воспользуемся стандартными средствами Maple. Вводим значения уравнение (1):

restart; with(PDEtools) : with(LinearAlgebra); DU1 := diff $(y(x), x, x)+\operatorname{lambda} \cdot y(x)=0$;

$$
\begin{aligned}
& D U 1:=\frac{\mathrm{d}^{2}}{\mathrm{~d} x^{2}} y(x)+\lambda y(x)=0 \\
& \text { Находим общее решение уравнения } D U \text { : } \\
& y:=\text { dsolve }(D U, y(x)) ; \text { dsolve }(D U, y(x)) ; y:=\operatorname{unapply}\left(\operatorname{rhs}\left(\frac{\circ}{\circ}\right), x\right) \text {; } \\
& Y:=y(x)={ }_{-} C 1 \sin (\sqrt{\lambda} x){ }_{-} C 2 \cos (\sqrt{\lambda} x) \\
& y(x)={ }_{-} C 1 \sin (\sqrt{\lambda} x)+{ }_{-} C 2 \cos (\sqrt{\lambda} x) \\
& y:=x \rightarrow{ }_{-} C 1 \sin (\sqrt{\lambda} x)+{ }_{-} C 2 \cos (\sqrt{\lambda} x)
\end{aligned}
$$

Вводим граничные условия 1-го рода:

$$
\text { assume }(b>a) ; \quad g 1:=y(a)=0 ; g 2:=y(b)=0 ;
$$

$$
\begin{aligned}
& g 1:={ }_{-} C 1 \sin (\sqrt{\lambda} a \sim)+{ }_{-} C 2 \cos (\sqrt{\lambda} a \sim)=0 \\
& g 2:={ }_{-} C 1 \sin (\sqrt{\lambda} b \sim)+{ }_{-} C 2 \cos (\sqrt{\lambda} b \sim)=0
\end{aligned}
$$

Сформируем систему для граничных условий, неизвестными которой являются коэффициенты_C1,_C2 :

sys $:=\{g 1, g 2\} ;$

sys $:=\left\{{ }_{-} C 1 \sin (\sqrt{\lambda} a \sim)+{ }_{-} C 2 \cos (\sqrt{\lambda} a \sim)=0,{ }_{-} C 1 \mathrm{si}\right.$

Затем составляем матрицу этой системы, используя команду GenerateMarix, которая формирует матрицу из коэффициентов уравнений системы:

$$
\begin{aligned}
& \mathrm{G} 1:=\text { GenerateMatrix(sys, [_C1, _C2 ]) ; } \\
& G 1:=\left[\begin{array}{cc}
\sin (\sqrt{\lambda} a \sim) & 0 \\
\sin \left(\sqrt{\lambda} b_{\sim}\right) & 0
\end{array}\right],\left[\begin{array}{l}
-C 2 \cos (\sqrt{\lambda} a \sim) \\
-C C 2 \cos (\sqrt{\lambda} b \sim)
\end{array}\right] \\
& G 12:=\left[\begin{array}{cccccc}
\sin (\sqrt{\lambda} a \sim) & 0 & -{ }_{-} C 2 \cos (\sqrt{\lambda} a \sim) & \cos (\sqrt{\lambda} a \sim) & 0 & -{ }_{-} C 1 \sin (\sqrt{\lambda} a \sim) \\
\sin (\sqrt{\lambda} b \sim) & 0 & -{ }_{-} C 2 \cos (\sqrt{\lambda} b \sim) & \cos (\sqrt{\lambda} b \sim) & 0 & -{ }_{-} C 1 \sin (\sqrt{\lambda} b \sim)
\end{array}\right] \\
& \mathrm{G} 2:=\text { GenerateMatrix(sys, [_C2, _C1]) ; } \\
& G 12:=\langle\langle G 1 \mid G 2\rangle\rangle \text {; } \\
& G 2:=\left[\begin{array}{cc}
\cos (\sqrt{\lambda} a \sim) & 0 \\
\cos (\sqrt{\lambda} b \sim) & 0
\end{array}\right],\left[\begin{array}{c}
{ }_{-} C 1 \sin (\sqrt{\lambda} a \sim) \\
{ }_{-} C 1 \sin (\sqrt{\lambda} b \sim)
\end{array}\right]
\end{aligned}
$$

По синтаксису команды, из 1-го уравнения системы должны быть выбраны коэффициенты при _ $C 1,{ }_{-} C 2$ и записаны в первую строку матрицы $G 1$. Аналогично, из 2-го уравнения во вторую строку. Вторая же матрица в строке , должна содержать свободные члены системы. Как видно, команда выполняется в Maple 17 своеобразно: из уравнений системы выбран командой коэффициент при _C1, затем свободный член . А вторая матрица содержит неизвестные _ $C 2$ со своими коэффициентами, которые перенесены вместо свободных членов. Для формирования требуемой матрицы системы, составляем матрицу $G 2$, затем соединяем матрицы: 


\begin{tabular}{|c|c|c|c|c|c|c|}
\hline \multirow{4}{*}{ Impact Factor: } & ISRA (India) & $=3.117$ & SIS (USA) & $=0.912$ & ICV (Poland) & $=6.630$ \\
\hline & ISI (Dubai, UAE & $=0.829$ & РИНЦ (Russia & $=0.156$ & PIF (India) & $=1.940$ \\
\hline & GIF (Australia) & $=0.564$ & ESJI (KZ) & $=8.716$ & IBI (India) & $=4.260$ \\
\hline & JIF & $=1.500$ & SJIF (Morocce & $=5.667$ & OAJI (USA) & $=0.350$ \\
\hline
\end{tabular}

Первый и третий столбцы матрицы $G 12$ и составляют матрицу коэффициентов при неизвестных _ $C 1,{ }_{-} C 2$ :

$$
\begin{gathered}
\text { G122 }:=\operatorname{Column}(G 12,1) \\
\text { G122 }:=\left[\begin{array}{c}
\sin (\sqrt{\lambda} a \sim) \\
\sin (\sqrt{\lambda} b \sim)
\end{array}\right] \\
G 123:=\left[\begin{array}{c}
\cos (\sqrt{\lambda} a \sim) \\
\cos (\sqrt{\lambda} b \sim)
\end{array}\right] \\
G:=\left[\begin{array}{ll}
\sin (\sqrt{\lambda} a \sim) & \cos (\sqrt{\lambda} a \sim) \\
\sin (\sqrt{\lambda} b \sim) & \cos (\sqrt{\lambda} b \sim)
\end{array}\right]
\end{gathered}
$$

Для нахождения $\lambda$ составляем матрицу и вычисляем определитель этой матрицы:

$$
\begin{gathered}
\text { deI }:=\text { combine }(\text { Determinant }(G)) ; \\
\text { del }:=\sin (\sqrt{\lambda} a \sim-\sqrt{\lambda} b \sim) .
\end{gathered}
$$

Как видим, определитель содержит тригонометрическую функцию. С помощью команды select выделяем нужное выражение в $d e l$. Для получения всех решений тригонометрического уравнения, которое представляет детерминант задаем значение глобальной переменной__EnvAllSolutions равным true, при этом вводится целочисленная системная переменная _Z1-:

$$
\begin{aligned}
& \text { del := select(has, del, lambda); } \\
& \text { EnvAllsolutions := true : } \\
& \text { lambda := solve(del, lambda); EnvAlISolutions:= true: } \\
& \begin{array}{c}
\text { del }:=\sin (\sqrt{\lambda} a \sim-\sqrt{\lambda} b \sim) \\
\lambda:=\frac{\pi^{2} Z_{1} \sim^{2}}{(-b \sim+a \sim)^{2}}
\end{array} \\
& \text { lambda := subs(_z1=k, lambda); } \\
& \lambda:=\frac{\pi^{2} k^{2}}{(-b \sim+a \sim)^{2}} .
\end{aligned}
$$

Находим $\lambda$ :
Теперь находим собственные функции: assume $(k$, posint $): y(x)$;

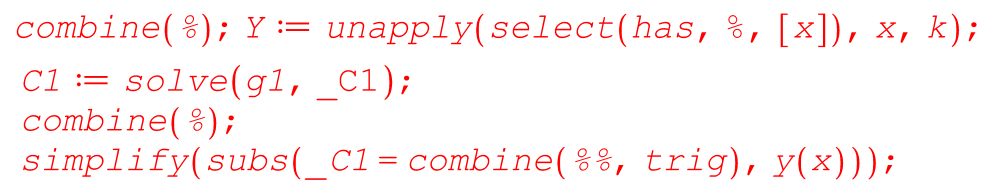

$$
\begin{gathered}
-C 1 \sin \left(\sqrt{\frac{\pi^{2} k^{2}}{(-b \sim+a \sim)^{2}}} x\right)+{ }_{-} C 2 \cos \left(\sqrt{\frac{\pi^{2} k \sim^{2}}{(-b \sim+a \sim)^{2}}} x\right) \\
C 1:=\frac{{ }_{-C 2 \cos \left(\frac{\pi k \sim a \sim}{-b \sim+a \sim}\right)}^{\sin \left(\frac{\pi k \sim a \sim}{-b \sim+a \sim}\right)}}{\frac{-C 2 \sin \left(\frac{\pi a \sim k \sim-\pi k \sim x}{-b \sim+a \sim}\right)}{\sin \left(\frac{\pi k \sim a \sim}{-b \sim+a \sim}\right)}} \\
\frac{-C 2 \cos \left(\frac{\pi k \sim a \sim}{-b \sim+a \sim}\right)}{\sin \left(\frac{\pi k \sim a \sim}{-b \sim+a \sim}\right)} \quad Y:=(x, k \sim) \rightarrow \sin \left(\frac{\pi a \sim k \sim-\pi k \sim x}{-b \sim+a \sim}\right) \\
\text { Иапользуем эту же программу для реше }
\end{gathered}
$$

Используем эту же программу для решения Задачи Штурма - Лиувилля с граничными условиями 2-го рода: 


\begin{tabular}{|c|c|c|c|c|c|c|}
\hline \multirow{4}{*}{ Impact Factor: } & ISRA (India) & $=3.117$ & SIS (USA) & $=0.912$ & ICV (Poland) & $=6.630$ \\
\hline & ISI (Dubai, UAE & $=0.829$ & РИНЦ (Russia & $=\mathbf{0 . 1 5 6}$ & PIF (India) & $=1.940$ \\
\hline & GIF (Australia) & $=0.564$ & ESJI (KZ) & $=8.716$ & IBI (India) & $=4.260$ \\
\hline & JIF & $=1.500$ & SJIF (Morocco & $=5.667$ & OAJI (USA) & $=0.350$ \\
\hline
\end{tabular}

$$
\begin{aligned}
&\left\{\begin{array}{l}
Y^{\prime \prime}(x)+\lambda Y(x)=0 \\
Y^{\prime}(a)=Y^{\prime}(b)=0
\end{array}\right. \begin{array}{l}
\text { тогда внесем изменения } \\
\text { условий: }
\end{array} \\
& g 1:=\mathrm{D}[1](y)(a)=0 ; g 2:=\mathrm{D}[1](y)(b)=0 ; \\
& g 1:={ }_{-} C 1 \cos (\sqrt{\lambda} a \sim) \sqrt{\lambda}-{ }_{-} C 2 \sin (\sqrt{\lambda} a \sim) \sqrt{\lambda}=0 \\
& g 2:={ }_{-} C 1 \cos (\sqrt{\lambda} b \sim) \sqrt{\lambda}{ }_{-} C 2 \sin (\sqrt{\lambda} b \sim) \sqrt{\lambda}=0
\end{aligned}
$$

тогда внесем изменения при введении граничных

\section{Conclusion}

Детерминат матрицы системы имеет вид:

$$
d e l:=\lambda \sin (\sqrt{\lambda} a \sim-\sqrt{\lambda} b \sim)
$$

и решая его, относительно $\lambda$, имеем:

$$
\lambda:=0, \frac{\pi^{2} Z Z 1 \sim^{2}}{(-b \sim+a \sim)^{2}} .
$$

Разобьем значения $\lambda$, принимая для решения сначала первое, а затем второе значение $\lambda$, получим 2 решения. Для ненулевого значения $\lambda$, имеем:

$$
\text { lambda := lambda[2]; }
$$

$$
\begin{gathered}
\lambda:=\frac{\pi^{2} Z Z 1 \sim^{2}}{(-b \sim+a \sim)^{2}} \\
Y:=(x, k \sim) \rightarrow \cos \left(\frac{\pi a \sim k \sim-\pi k \sim x}{-b \sim+a \sim}\right) .
\end{gathered}
$$

Аналогично, поступаем для $\lambda=0$. Описываемые программы применяются для определенных значений $a$ и $b$, для чего в начале программы вводятся их определенные значения.

\section{References:}

1. Bitsadze, A. V. (1982). Uravneniya matematicheskoy fiziki. (p.336). Moscow: Nauka.

2. Vladimirov, V. S. (1981). Uravneniya matematicheskoy fiziki. (p.512) Moscow: Nauka.

3. Mikhaylov, V. P. (1983). Differentsial'nye uravneniya s chastnymi proizvodnymi. (p.424). Moscow: Nauka.

4. Goloskokov, D. P. (2004). Uravneniya matematicheskoy fiziki. Reshenie zadach $v$ sisteme Maple uchebnik dlya vuzov. (p.539). $\mathrm{SPb}$.: Piter.

5. D'yakonov, V. P. (2006). Maple 9.5/10 v matematike, fizike i obrazovanii Izd: Piter

6. Enns, R. H., \& McGuire, G. C. (n.d.). Nonlinear Physics With Maple for Scientists and Engineers. ISBN 0-8176-4119-X
7. Davis, J. H. (n.d.). Differential Equations With Maple: An Interactive Approach. ISBN 0-81764181-5

8. Abell, M. L., \& Braselton, J. P. (n.d.). Differential Equations with Maple V. ISBN 012-041560-7

9. Franco Vivaldi (n.d.). Experimental Mathematics with Maple. ISBN 1-58488-233-6

10. Greene, R. L. (n.d.). Classical Mechanics With Maple. ISBN 0-387-94512-1

11. Lynch, S. (n.d.). Dynamical Systems with Applications using Maple. ISBN 0-8176-4150-5

12. Putz, J. F. (2003). Maple Animation. ISBN 158488-378-2 